\title{
Application of a Mesh Free Monte-Carlo Method to the Analysis of Dielectric Slabs in Electromagnetics
}

\author{
M. A. Ribeiro ${ }^{1,2, *}$, J. P. Pavia ${ }^{1,2}$, and N. Souto ${ }^{1,2}$ \\ ${ }^{1}$ University Institute of Lisbon - ISCTE-IUL, Av. das Forças Armadas, 1649-026 Lisbon, Portugal \\ ${ }^{2}$ Instituto de Telecomunicações, Av. Rovisco Pais, 1049-001 Lisbon, Portugal \\ *marco.ribeiro@iscte-iul.pt
}

\begin{abstract}
In this paper, we present a probabilistic MonteCarlo method to simulate the electromagnetic field in multiple interface problems based on transmission lines. We present numerical results for the simplest case of the propagation of gaussian pulses and sinusoidal sources in lossy and lossless dielectric slabs which demonstrate the applicability of the method to solve network problem in microwave theory. We also present a methodology to obtain the amplitude and phase associated with every point in the domain without resorting to fourier transformations. This method is an important achievement in the development of our Monte-Carlo method because otherwise we would have to integrate the field over time to achieve similar results, which would be unpractical for a Monte-Carlo method. Finally, we compare our results with well established theory to validate the methods.
\end{abstract}

Index Terms-Monte-Carlo methods, Electromagnetics, Random Walks, Microwave theory.

\section{INTRODUCTION}

Numerical methods have been applied for many years in computational electromagnetics to overcome the inability to derive closed form solutions of Maxwell's equations in realworld problems related to scattering, radiation and material characterization. The finite difference time domain method (FDTD), the finite element method (FEM) and the method of moments (MoM) are the three preferred numerical techniques for the simulation of Maxwell's equations. All of these methods have one thing in common: they use a grid to store the electromagnetic fields. Methods that resort to a grid to represent all or part of its domain have important limitations such as numerical dispersion, instability and convergence issues. Usually when solving hyperbolic PDEs numerically it is necessary to impose constraints, such as the CourantFriedrichs-Lewy (CFL) condition, to ensure convergence [1]. These constraints usually imply that we cannot simulate large structures at high frequencies without scaling the problem to unpractical limits. Grid-less methods can easily overcome this issue if time is also not discretized because they do not have to comply with the CFL condition [2]. These methods are also particularly versatile in dealing with open boundaries and memory resources since we only need to keep track of the fields at points where we want to calculate the fields [3]. This is often considered a disadvantage if the electromagnetic field is to be known at all points in the domain, [4], but grid-based methods that discretize the entire domain into memory, like the FDTD and FEM methods, cannot support open boundaries. Hence, FDTD and FEM have to resort to absorbing materials, such as the perfectly matched layer (PML), to deal with undesired boundary reflections [5]. These PMLs are very efficient impedance matched materials but will always introduce some artifacts in the simulation domain.

The modeling of materials and boundaries is also constrained to the shape of the grid. Grid-less method, like the floating random walk that is employed to solve static electric fields, can model material geometries and boundaries and inhomogeneous materials very accurately [6], [7]. The FDTD method, on the other hand, has the important limitation that it uses a leapfrog grid to lay out the fields in space in a geometric pattern called Yee grid. This is a rectangular grid and so materials and boundaries in FDTD are constrained to rectangular shapes. The FEM can handle complicated geometries/boundaries with relative ease because it allows the grid points to fit materials and boundaries. FEM is thus the method of choice in all types of electromagnetic simulations involving complicated geometries [8].

Finally, Grid-less methods are especially suited for parallel computing since the in-existence of a grid normally implies little communication between parallel thread. If a grid exists, such as in classical methods, then parallel processes have to exchange information related to the solution of fields in the domain boundary that separates two neighboring threads. For example, with the grid-less Monte-Carlo method to be discussed in this paper we simply write a program that calculates the fields at a given point in space at a given time and it gets automatically distributed across hundreds of cores for thousands of threads.

In this paper we apply the Monte-Carlo method developed in [2] to the analysis of wave propagation in dielectric slabs with and without losses. We perform simulations to read out reflection and transmission data that we can compare to theory in order to corroborate the veracity of the method. The article also propose a new technique to extract frequency information from the simulations without resorting to Fourier transformation. The article is organized as follows. In section II we present the theoretical details that lay the foundations for our monte-carlo method (see [2] for a more detailed discussion). In section III, we explain how network components are interconnected to form the simulation system. This involves 
the development of boundary conditions following closely the methodology and techniques outlined in network theory, [9]. In section IV we present our numerical results, followed by well-founded theoretical calculations, to corroborate the methodologies presented. Finally, in section V, we draw the conclusions.

\section{Probabilistic Formulation of the Lossy Line EQUATIONS}

An electromagnetic model of a general two-conductor uniform transmission line must take into account a number of factors in order to emulate the physical behavior of the line in a realistic manner. The three most important features are the capacitive and inductive energy storage characteristics of the line, the conductivity of the wires and the losses in the dielectric medium. For harmonic fields these effects are accurately modeled by the following time-harmonic transmissionline equations:

$$
\begin{aligned}
\frac{\partial}{\partial t}\left(\begin{array}{c}
v(x, t) \\
i(x, t)
\end{array}\right)= & -\mathbf{M} \frac{\partial}{\partial x}\left(\begin{array}{c}
v(x, t) \\
i(x, t)
\end{array}\right) \\
& -\mathbf{N}\left(\begin{array}{c}
v(x, t) \\
i(x, t)
\end{array}\right)
\end{aligned}
$$

where

$$
\mathbf{M}=\left(\begin{array}{cc}
0 & 1 / C \\
1 / L & 0
\end{array}\right), \quad \mathbf{N}=\left(\begin{array}{cc}
G / C & 0 \\
0 & R / L
\end{array}\right)
$$

We rewrite these equations as a superposition of forward $f(x, t)$ and backward $b(x, t)$ terms as follows, [2]:

$$
\begin{aligned}
v(x, t) & =f(x, t)+b(x, t) \\
i(x, t) & =\frac{1}{R_{0}}(f(x, t)-b(x, t))
\end{aligned}
$$

where $R_{0}=\sqrt{L / C}$. It then follows from the system equations above that

$$
\begin{aligned}
\frac{\partial f(x, t)}{\partial t} & =-c_{0} \frac{\partial f(x, t)}{\partial x}-(\lambda+\kappa) f(x, t) \\
& +\lambda b(x, t) \\
\frac{\partial b(x, t)}{\partial t} & =c_{0} \frac{\partial b(x, t)}{\partial x}+\lambda f(x, t) \\
& -(\lambda+\kappa) b(x, t)
\end{aligned}
$$

where $c_{0}=1 / \sqrt{L C}$. The solution of these equations at time instant $t_{n}$ in a generic point of the transmission line $x_{n}$ is given by

$$
\begin{aligned}
\tilde{f}\left(x_{n}, t_{n}\right) & =\tilde{f}\left(x_{n}-c_{0} t_{1}, t_{n}-t_{1}\right) \\
& +\lambda \int_{0}^{t_{1}} \tilde{b}\left(x_{n}-c_{0} s, t_{n}-s\right) d s \\
\tilde{b}\left(x_{n}, t_{n}\right) & =\tilde{b}\left(x_{n}+c_{0} t_{2}, t_{n}-t_{2}\right) \\
& +\lambda \int_{0}^{t_{2}} \tilde{f}\left(x_{n}+c_{0} s, t_{n}-s\right) d s
\end{aligned}
$$

where we have introduced the transformation

$$
\tilde{f}(x, t)=f(x, t) e^{(\lambda+\kappa) t}, \quad \tilde{b}(x, t)=b(x, t) e^{(\lambda+\kappa) t}
$$

and the parameters

$$
\lambda=\frac{1}{2}\left(\frac{R}{L}-\frac{G}{C}\right), \quad \kappa=\frac{G}{C}
$$

The probabilistic method then results from the introduction of the exponential distribution

$$
p(s)=(\lambda+\kappa) e^{-(\lambda+\kappa) s} H(s)
$$

which lets us write the previous equations in terms of expected values:

$$
\begin{aligned}
f\left(x_{n}, t_{n}\right) & =E\left[f\left(x_{n}-c_{0} t_{1}, t_{n}-t_{1}\right) H\left(s-t_{1}\right)\right. \\
& \left.+\frac{\lambda}{\lambda+\kappa} b\left(x_{n}-c_{0} s, t_{n}-s\right) H\left(t_{1}-s\right)\right] \\
b\left(x_{n}, t_{n}\right) & =E\left[b\left(x_{n}+c_{0} t_{2}, t_{n}-t_{2}\right) H\left(s-t_{2}\right)\right. \\
& \left.+\frac{\lambda}{\lambda+\kappa} f\left(x_{n}+c_{0} s, t_{n}-s\right) H\left(t_{2}-s\right)\right]
\end{aligned}
$$

In these expression $H(s)$ is the Heaviside function. To evaluate $f\left(x_{n}, t_{n}\right)$ and $b\left(x_{n}, t_{n}\right)$ we must introduce the summations

$$
\begin{aligned}
& f\left(x_{n}, t_{n}\right) \simeq \frac{1}{N} \sum_{k=1}^{N} \bar{f}\left(x_{n}, t_{n}\right) \\
& b\left(x_{n}, t_{n}\right) \simeq \frac{1}{N} \sum_{k=1}^{N} \bar{b}\left(x_{n}, t_{n}\right)
\end{aligned}
$$

where $N$ represents the number of independent samples that we wish to compute. To compute a single trial of $\bar{f}\left(x_{n}, t_{n}\right)$ we generate a random time $s$ using the exponential distribution given above. Depending on the value of $s$ one of the heaviside terms in (10) will yield one while the other will give zero. If $H\left(s-t_{1}\right)=1$ we proceed by setting $\bar{f}\left(x_{n}, t_{n}\right)=f\left(x_{n}-c_{0} t_{1}, t_{n}-t_{1}\right)$. Otherwise, we shall make $\bar{f}\left(x_{n}, t_{n}\right)=\lambda /(\lambda+\kappa) b\left(x_{n}-c_{0} s, t_{n}-s\right)$. The problem is now to find $b$ or $f$ in a previous time and this is done exactly in the same manner by generating another random time $s$. The process will stop when the generated trajectory colides with initial conditions. We repeat the process $N$ times and use (11) to estimate $f\left(x_{n}, t_{n}\right)$.

\section{PRobabilistic FORMUlation OF Boundary CONDitions: ANALYSis Of A LOSSY Dielectric Slab}

Up until now, we have only described the transmission line as a general component to which we have not connected anything. If the line interfaces with other components, trajectories can collide with the boundaries, which in turn will contribute with new equations that must be fulfilled in order to find the field solutions. In this section we will show how we can use the method outlined above to simulate the propagation of electromagnetic waves in the dielectric slab presented in Fig. 1. The conditions for the generator and the boundaries between transmission lines are

$$
\begin{gathered}
f_{1}(0, t)=\zeta v_{g}(t)+\Gamma_{g} b(0, t), \\
b_{1}\left(l_{1}, t\right)=\tau_{21} b_{2}(0, t)+\Gamma_{12} f_{1}\left(l_{1}, t\right) \\
f_{2}(0, t)=\tau_{12} f_{1}\left(l_{1}, t\right)+\Gamma_{21} b_{2}(0, t),
\end{gathered}
$$




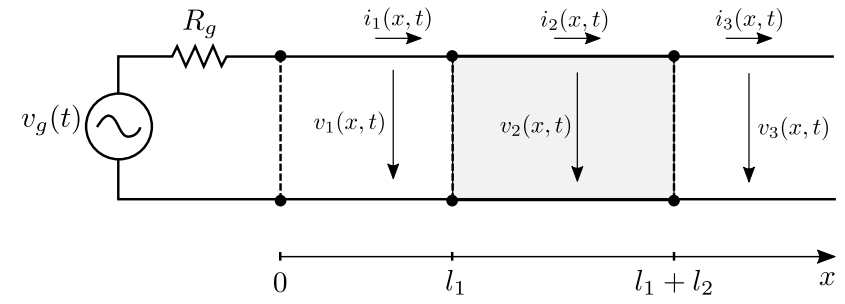

Fig. 1. A lossy dielectric slab terminated at one end by a voltage source. The other end is left open.

$$
\begin{aligned}
& b_{2}\left(l_{2}, t\right)=\tau_{31} b_{3}(0, t)+\Gamma_{23} f_{2}\left(l_{2}, t\right) \\
& f_{3}(0, t)=\tau_{23} f_{2}\left(l_{2}, t\right)+\Gamma_{32} b_{3}(0, t)
\end{aligned}
$$

where $\zeta$ is the voltage divider coefficient and $\Gamma_{g}$ is the reflection coefficient at the source

$$
\zeta=\frac{R_{01}}{R_{01}+R_{g}}, \quad \Gamma_{g}=\frac{R_{g}-R_{01}}{R_{g}+R_{01}}
$$

$\Gamma_{i j}$ and $\tau_{i j}$ are, respectively, the reflections and transmission coefficients from line $i$ to line $j$, which are given by

$$
\Gamma_{i j}=\frac{R_{0 j}-R_{0 i}}{R_{0 j}+R_{0 i}}, \quad \tau_{i j}=\frac{2 R_{0 j}}{R_{0 i}+R_{0 j}} .
$$

In addition to the boundary conditions we must also take into account the initial conditions. In our case we will simply set $f_{i}(x, 0)=0$ and $g_{i}(x, 0)=0$ with $i=1,2,3$, which corresponds to having the voltage and current equal to zero in $x \in[0, \infty[$. Note that we deliberately introduced an open boundary in the right end of the simulation.

For the source we will consider two examples: A Gaussian source of amplitude $A=2$, internal resistance $R_{g}=R_{1}$ (matched generator) and spread $s_{0}$, which takes its maximum value at time instant $t=t_{0}>0$. The expression for this source is

$$
v_{g}(t)=A \exp \left[-\frac{1}{2}\left(\frac{t-t_{0}}{s_{0}}\right)^{2}\right]
$$

We also consider a sinusoidal source of unit amplitude and frequency $f_{0}, v_{g}(t)=\sin \left(2 \pi f_{0} t\right)$. With this source we shall infer the amplitude and phase associated with each point of the structure, at frequency $f_{0}$. To achieve this we simply calculate, at each point, the value of a field quantity, lets say the voltage $v$, at a given instant $t_{i}$ and at $t_{f}=t_{i}+T_{0} / 4$, where $T_{0}=1 / f_{0}$ is the period. Then the amplitude and phase associated with the point are given by

$$
\begin{gathered}
|V(x)|=\sqrt{v_{i}^{2}(x)+v_{f}^{2}(x)} \\
\arg V(x)=-\arctan \left(\frac{v_{f}(x)}{v_{i}(x)}\right)
\end{gathered}
$$

where $V(x)$ is the complex coefficient of the fourier transform of $v(x, t), v_{i}=v\left(x, t_{i}\right)$ and $v_{f}=v\left(x, t_{f}\right)$.

\section{Numerical Results}

In this section we present numerical simulations that corroborate the operation of the probabilistic method. Let us consider that lines 1 and 3 are both lossless with lengths $l_{1}=l_{2}=1 \mathrm{~m}$ and parameters $L_{1}=L_{3}=4 \pi \times 10^{-7}$ $\mathrm{H} / \mathrm{m}, C_{1}=C_{3}=8.85 \times 10^{-12} \mathrm{~F} / \mathrm{m}, R_{1}=R_{3}=0$ and $G_{1}=G_{3}=0$. For line 2 we use $L_{2}=L_{1}, C_{2}=4 L_{1}$. In Figure 2 we present the amplitude and phase associate with points between $x=0$ and $x=2.8 \mathrm{~m}$ for a frequency of $500 \mathrm{Mhz}$. In this simulation, we have assumed that line 2 is also lossless for simplicity. The Monte-Carlo parameter was set to $N=10^{3}$. From this figure we where able to measure the values of the reflection coefficient at the left interface $\Gamma$ and the transmission coefficient $\tau$ at the right interface. We found $|\tau|=0.846,1+|\Gamma|=1.55,1-|\Gamma|=0.45$, yielding $|\Gamma|=0.55$. The theoretical expressions yield

$$
\begin{gathered}
\Gamma=\frac{\Gamma_{12}+\Gamma_{23} e^{-2 j k_{1} l_{2}}}{1+\Gamma_{12} \Gamma_{23} e^{-2 j k_{1} l_{2}}} \quad \mapsto \quad|\Gamma|=0.543 \\
\tau=\frac{\tau_{12} \tau_{23} e^{-j k_{1} l_{2}}}{1+\Gamma_{12} \Gamma_{23} e^{-2 j k_{1} l_{1}}} \quad \mapsto \quad|\tau|=0.84
\end{gathered}
$$

in excellent agreement with the simulation results. In Fig. 3
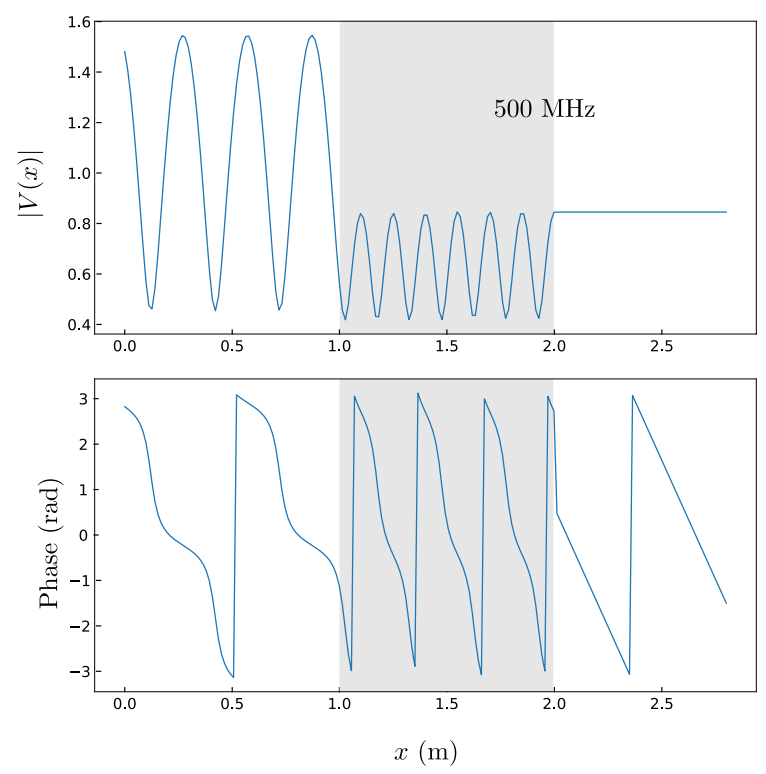

Fig. 2. Amplitude and phase information obtain with the Monte-Carlo method for a dielectric slab illuminated at $500 \mathrm{MHz}$.

we show the results of a Gaussian pulse with $t_{0}=0.83 \mathrm{~ns}$ and $s_{0}=0.16 \mathrm{~ns}$ as it propagates through space and interacts with the slab. The figure registers the fields $v(x, t)$ and $R_{0} i(x, t)$ at point $x=0.5 \mathrm{~m}$ until $t=22 \mathrm{~ns}$. Fig. 3a) is for the lossless case $\left(R_{2}=0\right)$, Fig. $\left.3 \mathrm{~b}\right)$ for $R_{2}=200 \Omega / \mathrm{m}$ and Fig. $\left.3 \mathrm{c}\right)$ for $400 \Omega / \mathrm{m}$. In all three figures we can see first the pulse that started at the generator and passes at $x=0.5$ towards the slab. Then, the pulse is partially reflected at the left boundary of the slab and passes again at $x=0.5$ towards the generator, where it is absorbed. The voltage amplitude of this pulse is given 
by $\Gamma_{12}=-0.33$ and, as can be seen, is sensibly the same in the three cases because this pulse did not travel in the lossy material. Finally, the third pulse that we can see in all three figures with different amplitudes is the pulse that was reflected at the right boundary of the slab and was then transmitted to line 1 at the first interface. It passes at $x=0.5$ and is then absorbed at the generator. We see then that the Monte Carlo method was able to predict the attenuation due to losses in line 2 .
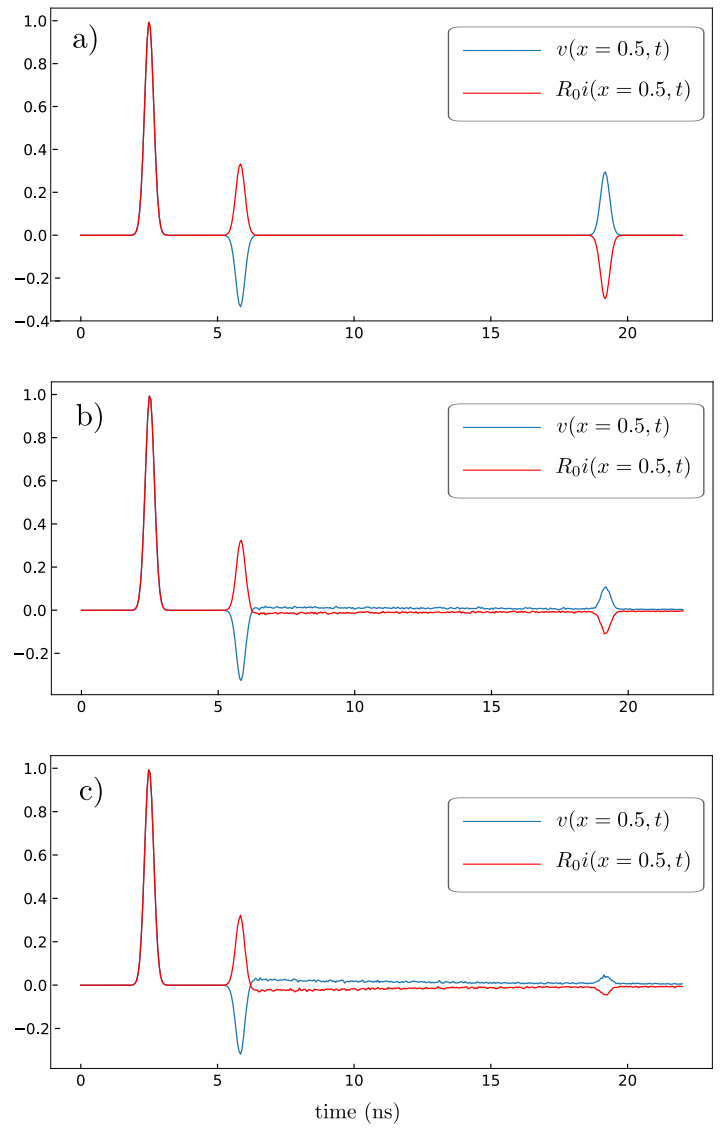

Fig. 3. Amplitude and phase information obtain with the Monte-Carlo method for a dielectric slab illuminated at $500 \mathrm{MHz}$.

\section{Conclusions}

In this paper we studied the problem of electromagnetic wave propagation in the presence of a material slab using a novel Monte-Carlo method for electromagnetics. Lossy and lossless structures were considered to test the method. We have also shown how we can render frequency information, such as amplitude and phase at a given frequency for all points in the domain, without resorting to the conventional methods using the Fourier transform. This has the obvious advantage that it is not necessary to integrate the fields over time, which would be an impractical task for the method proposed here. We conclude that the developed method offers many potentialities, although not a substitute for the classical methods such as the FDTD, FEM and MoM. In fact the main strengths of the method come form the fact that it is grid-less. Since it is a monte-carlo method, its convergence is slow and time is required to obtain accurate results. The generalization to higher dimensions, and the possibility of tackling more complex geometries and materials will be the subject of future research of the authors.

\section{ACKNOWLEDGMENT}

The authors acknowledge financial support by Fundação para a Ciência e a Tecnologia under the project TUBITAK/0002/2014.

\section{REFERENCES}

[1] A. Taflove and S. C. Hagness, Computational Electrodynamics: Computational Electrodynamics: The Finite-Difference Time-Domain Method, 2nd ed., Artech House, 2000.

[2] J.A. Acebron and M. A. Ribeiro, "A Monte Carlo method for solving the one-dimensional telegraph equations with boundary conditions," Journal of Computational Physics, vol 305, pp. 29-43, 2016.

[3] G. Ala, E. Francomano, A. Tortorici, E. Toscano and F. Viola, "Smoothed Particle ElectroMagnetics: A mesh-free solver for transients," Journal of Computational and Applied Mathematics, vol. 191, pp. 194 205, 2006.

[4] G. E. Zinsmeiter and S. S. Pan, "A method for improving the efficiency of Monte Carlo calculation of heat conduction problems," J. Heat Transfer, Trans. ASME, Series C, vol. 96, pp. 246-248, 1974.

[5] Berenger, J. P., "A Perfectly Matched Layer for the Absorption of Electromagnetic Waves," Journal of Computational Physics, vol. 114, pp. 185-200, 1994.

[6] R. Schlott, "A Monte Carlo method for the Dirichlet problem of dielectric edges," IEEE Trans. Micro. Theo. Tech., vol. 36, no. 4, pp. 724-730, 1988.

[7] J. N. Jere and Y.L.L. Coz, "An improved floating-random-walk algorithm for solving multi-dielectric Dirichlet problems," IEEE Trans. Micro. Theo. Tech., vol. 41, no. 2, pp. 252-329, 1993.

[8] M. N.O. Sadiku, Numerical Techniques in Electromagnetics, 2nd ed., CRC Press, 2000.

[9] D. M. Pozar, Microwave Engineering, 2nd ed., Wiley, 1998, pp.182-250. 